\title{
SMART TRAVELLING WITH RADIO FREQUENCY IDENTIFICATION
}

\author{
${ }^{1}$ Zainab Rasheed Fahad Mirza and ${ }^{2}$ Muhammad Nawaz Brohi \\ ${ }^{1}$ Department of Research and Development Al Madad Eng. Services Ajman, UAE \\ ${ }^{2}$ Department of Computing, Shaheed Zulfiqar Ali Bhutto Institute of Science and Technology (SZABIST) Dubai, UAE
}

Received 2013-09-09, Revised 2013-10-11; Accepted 2013-10-29

\begin{abstract}
Radio Frequency Identification (RFID) technology is being widely used now-a-days and is becoming more popular with every passing day. There are varied applications of this technology in various fields such as industry, communication, travel and transportation. Roads, rail ways, air traffic and container vessel shipping all share underlying abstractions of transportation nets with hubs. This study is concerned with applications of RFID technology with Cloud computing, innovation in the field of travelling and its applications for different modes of transportation at Harbors, airports, train stations, road intersections providing security, coordination, to the users. Combined with other technologies, RFID technology is being used for modern airports baggage system, for railroad car identification, in container transportation systems, for fuel supply control of vehicles, in traffic management system and in travelling information system.
\end{abstract}

Keywords: FRAM, Cloud RFID, ETC Transponders, FSC, Intelligent Traffic Control

\section{INTRODUCTION}

RFID technology provides innovative services in transportation allowing users to make smarter use of transport systems. Various combination of Information technologies with RFID are a applied in the field of transport, including infrastructure, vehicles and users and in traffic management and mobility management and as an interface for other modes of transport. In the fast paced developing world many areas are being urbanized resulting in increased congestion in multimodal transportation system which includes walking, bicycle transport, motorcycle, busses and trains, other than personal vehicles. Communication cooperation on road is mainly of two types, vehicle to vehicle and vehicle to infrastructure which can be very helpful to provide safe, secure and comfortable journey to passengers. Among many methods RFID is very efficient to allow wireless communication among vehicles and infrastructure. Cloud computing allows RFID technology to be more scalable and reliable. Data collected from RFID needs faster processing, efficient results and reliable storage systems provided by cloud Corresponding Author: Zainab Rasheed Fahad Mirza, Department of Research and Development Al Madad Eng. Services Ajman, UAE computing. Cloud RFID can be used as middle ware or interface between various transport control process having implemented RFID technology and backend global data resources.

Various forms of wireless communications technologies have been proposed for intelligent transportation systems but the most competent and successful implementation is for RFID technology. One of the cheap and most used method for collecting floating car data e.g., location, time, speed is using RFID technology. Various detectors are mounted across the roads which detect unique RFID tag number attached in each car. Calculating the detection time and speed of moving vehicle between two detectors helps capturing data. These detectors can also work as ETC electronic toll collection transponders been implemented in many areas of world to manage traffic congestions. In Dubai active RFID tags mounted on the rear chassis of 12000 trucks carrying cargo to and from Jebel Ali port to speed up vehicle entry and exit and increase security. RFID technology has saved most of the paperwork involved before and an average of $10 \mathrm{~min}$ is saved in checking out process of each truck (Wessel, 2009). 


\subsection{RFID Communication for Transport Systems}

\subsubsection{Asynchronous Communication for Online and Offline Use}

While many mobile devices are equipped with the ability to communicate over a Wi-Fi network, applications should be built with the assumptions that the network may not always be available or that it may not make a sense to constantly communicate over the network. Mobile devices that use wide area wireless cellular network need to be aware of network limitations certain issues such as limited, expensive bandwidth and battery life are such that only the most important information can be sent over the network when it is available. RFID solutions involving mobile devices should not depend on constant connection between device and server. Instead mobile device should be able to do functions offline as much as possible. Mobile application should be able to collect data enable decision making and general valuable information offline. This valuable information can be send to business logic at the edge of network running on the PC or server for further processing and integration in to the enterprise. Two common models for asynchronous communication are database synchronization where a mobile application interacts with a local relational database with changed synchronized between it and consolidated database and direct communication to the server (Want, 2006).

\subsection{Communication Capabilities of RFID}

With its communication capabilities RFID can provide real time tracking to allow for the maximum data availability and accessibility. By integrating with common industrial networking protocols, such as profibus Decentralized Peripherals (DP), device net and industrial Ethernet, RFID allows manufactures to optimize data management (Want, 2006). RFID data provides a large quantity of read or write operations with high speed ultimately increasing tracking capabilities to improve operations. RFID offers increased memory storage over barcode options with Ferroelectric Random Access Memory (FRAM) a nonvolatile memory device that can hold written data even after it is powered off. FRAM data carries processing data up to ten times faster, enabling it to withstand more operations, some even up to one billion cycles.

RFID's ability to monitor objects so closely without the need of halting the movement eliminates the risk of error during the identification of fast speed moving objects. By tracking one object through the entire transport process, it can be easily and quickly identify if any object or multiple objects had any mishandling. Further, system can store detailed information about a each moving object.

While barcode technology offers read only solution, RFID can provide users with read and write flexibility. This allows information to be added/edited on the tag or in database storing tag information which allows processing while object are on move rather than having to include a new tag for each individual variable.

\subsection{RFID in Transport System Applications}

\subsubsection{RFID Technology for Airports Baggage System (ABS)}

One form of RFID technology that has been deployed is at US Airways and Alaska Airlines (Slawsky, 2011). Invisible RFID chips are now being placed on baggage tags while they are printed. Inside the baggage handling system, the tags are scanned, allowing the handlers to send the bags to the correct destination.

Why is RFID advantageous in this situation? Most airports use standard barcode scanners, while one RFID reader can read the tag wherever it is located on the bag (Slawsky, 2011). With a barcode scanner, an airport needs to place four to six of them at any scanning point so they can cover any possible angle on the bag as direct line of sight is required. Weather resistant kiosks are installed where passengers tag their own bags. Kiosks can handle 55 customer per hour compared to 21 customers with live attendants for baggage check in. The result of using RFID is that fewer scanners need to be purchased, saving the airport money and the technology allows the bags to move through the system faster, preventing congestion and delays.

\subsection{RFID Technology and Container Transportation System}

The container transportation with the characteristics of highly effective, convenient and safe has been the important form of transportation modernization. With the rapid development of our economy, the harbor becomes an important point of our country modern logistics and supply chain. At present container number recognition technology always uses the number image recognition but the accuracy rate is only about $90 \%$ (Huang et al., 2011). It is easily influenced by weather conditions. Furthermore collect container information through the manual, operations some information can't meet the real time and accurate requirements, impacting the modern logistics, the cargo allocation benefit and management 
level of supply chain. The application of RFID technology on container that is the appearance of container electronic tag system, can improve above problems effectively. The using of container electronic tag auto-recognition technology, is not only enhance the speed of container passing the harbor but also unify and standardize the information management of domestic trade container, overcoming the low information accuracy rate, incomplete information.

\subsection{RFID Technology and Fuel Supply Control (FSC) of Vehicles}

Public transportation companies aim to incorporate quality and modern technologies on their services hoping to achieve the optimization of the employed material resources. Although available resources are controlled, there is always lack of reliability in the process involved. In modern times RFID technology is being used very effectively for fuel supply control of vehicles fleets. This system is based upon a modular and adaptive RFID middleware (cloud RFID) which surveys as a foundation to the development of RFID applications (Amaral et al., 2009).

A fuel supply operation consists in the approximation of the vehicle near to the fuel pump where a professional is designated to park the vehicle, enter the amount of liters planned and manually control the fuel supply activity. This procedure must be repeated for a fleet of approximately eighty vehicles, once or twice a day for thirty days a month.

For FSC of vehicles infrastructure deployed at the company consists of three fuel pumps, a RFID reader, three RFID antennas, a proprietary hardware module to control the pumps, a PC/Host TV hosts several applications and RFID tags one for each vehicle used. FSC process has been modified and automated, fuel pumps are blocked and unblocked by RFID system and Cloud RFID is a middle ware which integrates all FSC process (Amaral et al., 2009).

\subsection{RFID Technology and Vehicle Identification}

Automatic vehicle identification system is based upon RFID. For vehicle identification electronic tags are used when these vehicles travel along the road which was installed automatic car identification system, all kinds of vehicles information of car tag is read and transmitted. Major requirement are: passive tag, high speed reading, high reading range, high robustness and high temperature range. Classical RFID systems do not provide all these properties in combination for rail road car identification an RFID system on $2.45 \mathrm{GHz}$ based on Surface Acoustic Waves (SAW) is used (Li, 2010).
Automatic vehicle identification system based on RFID mainly installed in the on board electronic tags installed in the vehicle the base station of vehicle message identification established in particular location. The on board electronic tags are a data carrier of automatic vehicle identification system based on RFID technology, usually uses passive RFID tags working in UHF band.

\subsection{Traffic Management System and RFID}

Based on the theory of integration in system engineering intelligent transportation system is characterized by its modernization, accuracy, efficiency in a wider range and various directions. It involves advanced technology in information, computer data communication, sensor electronic control, automatic control operational research and artificial intelligence. Identification and location of vehicles can greatly improve the efficiency of road, constantly monitor road environment save operating consists (Guo et al., 2010). RFID technology can meet the requirements of vehicle location and status monitoring of traffic operation.

Traffic management system based on RFID technology, uses cluster nodes installed on both sides of road, these nodes from a self-organization network. The traffic information collection sensors communicate with each adjacent cluster. The final data is gathered to the gateway node. The gateway node can be used as a module installed in the intersection traffic controller. RFID tags and sensor nodes are independent to keep stored in the monitoring area. RFID reader is used for the base station. The sensor nodes send the data after its transfer to the local PC or remote network. RFID can be used for clarification of duty as an intelligent base station device, for RFID and data WSNL system integration. In the network, systems consist of five types of facilities:

- Base stations: They process data through a microprocessor

- Sensor nodess

- RFID tags: These tags store ID number, item type destination, transport properties etc.

- Device reader: It reads tag included in the item identification, information and environmental data.

- Center data platform: This is the core of storage system which includes daily information database.

\subsection{RFID and Travel Information}

In an Intelligent Transport System (ITS) communication system is highly dynamic and unstable. Due to high mobility and low duty cycle of communication link, the delay could vary provision of right information quickly without any delay is a key to 
intelligent transport system. However it is very expensive for fixed infrastructure. RFID enabled vehicular wireless query for travel information is an alternative which can leverage mobile nodes to bridge the gap between the information isolated islands often existing in mobile and wireless networks. Providing useful and timely travel information to passengers or travelers is very effective to eliminate unnecessary travel and optimize the traffics of transportation system. RFID technology can improve transportation security and safety (Luo et al., 2011).

\section{CONCLUSION}

This study discusses the technology feasibility and advantages of the transport application based on combination of Cloud RFID technology with various other IT technologies. Use of RFID technology in Transport and Travel sector has greatly promoted the development of intelligent transportation systems over heterogeneous adaptive network architectures. With Rapid development of transportation system many issues for more efficient and reliable monitoring and access control transport systems have come forward. Deployment of Cloud RFID as a middle ware along with RFID technology can transform current manual systems into requirements for respective transportation system monitoring and control systems.

This study concludes with the aim to develop right systems expected to be received by various transportation and traffic authorities.

\section{REFERENCES}

Amaral, L.A., F.P. Hessel, E.A. Bezerra1, J.C. Correa and O.B. Longhi et al., 2009. Using CloudRFID middleware for fuel supply control of vehicles fleets. Proceedings of the IEEE Conference on Emerging Technologies and Factory Automation, Sept. 22-25, IEEE Xplore Press, Mallorca, pp: 1-4. DOI: 10.1109/ETFA.2009.5347223
Guo, L., W. Fang, G. Wang and L. Zheng, 2010. Intelligent traffic management system base on WSN and RFID. Proceedings of the International Conference On Computer and Communication Technologies in Agriculture Engineering (CCTAE), Jun. 12-13. IEEE Xplore Press, Chengdu, pp: 227230. DOI: 10.1109/CCTAE.2010.5544797

Huang, X., G. Zhang, Q. Sun and D. Qing, 2011. Container transportation system using $2.45 \mathrm{GHz}$ active RFID technology. Proceedings of the International Conference on Remote Sensing, Environment and Transportation Engineering (RSETE), Jun. 24-26, IEEE Xplore Press, Nanjing, pp: 3030-3033. DOI: 10.1109/RSETE.2011.5964953

Li, C., 2010. Automatic Vehicle Identification (AVI) system based on RFID. Proceedings of the International Conference on Anti-Counterfeiting Security and Identification in Communication (ASID), Jul. 18-20, IEEE Xplore Press, Chengdu, pp: 281-284. DOI: 10.1109/ICASID.2010.5551336

Luo, Z., T. Zhang and C. Wang, 2011. RFID enabled vehicular wireless query for travel information in intelligent transportation system. Proceedings of the IEEE International Conference on RFIDTechnologies and Applications (RFID-TA), Sept. 15-16, IEEE Xplore Press, Sitges, pp: 415-420. DOI: 10.1109/RFID-TA.2011.6068671

Slawsky, R., 2011. Baggage self-check-in the latest airport innovation. RFID J.

Want, R., 2006. An introduction to RFID technology. IEEE Pervasive Comput., 5: 25-33. DOI: 10.1109/MPRV.2006.2

Wessel, R., 2009. DP world ramps up its Dubai deployment. RFID J. 